\title{
Paroxysmal atrial fibrillation developed during incomplete epidural anesthesia -A case report-
}

\author{
Min Je Choi, Hye Won Shin, Sung Uk Choi, Ji Yong Park, Hye Won Lee, Hae Ja Lim, Suk Min Yoon, \\ and Seong Ho Chang
}

Department of Anesthesiology and Pain Medicine, College of Medicine, Korea University, Seoul, Korea

Atrial fibrillation (AF) is the most common sustained tachyarrhythmia, and occurs in organic heart disease such as rheumatic, atherosclerotic and hypertensive heart disease. In recent studies, the sympathetic and parasympathetic nervous systems have been shown to have important roles in initiating paroxysmal AF. We report here a patient who developed paroxysmal AF that might be a result of an imbalance of the sympathetic-parasympathetic systems due to epidural anesthesia, and that was potentiated by pain with inadequate analgesia. A 69-year-old woman was scheduled for operation of a right-sided ankle fracture. Twenty minutes after epidural drug injection, paroxysmal AF occurred. Even after intravenous administration of esmolol and digoxin, AF continued. After transfer to the intensive care unit, her heart rate gradually decreased and AF disappeared. During perioperative anesthetic management, the proper preoperative prevention and intraoperative treatment are needed in AF high-risk patients. (Korean J Anesthesiol 2010; 59: S58-S61)

Key Words: Atrial fibrillation, Autonomic nervous system, Epidural anesthesia.

Atrial fibrillation (AF) is a common cardiac arrhythmia and is characterized by rapid atrial contraction and minimal mechanical activity. Although it is not immediately life threatening, AF can cause troublesome symptoms and poses a risk of stroke. Most of AF is associated with old age, cardiopulmonary disease, and endocrine abnormalities [1], but some instances of $\mathrm{AF}$ are associated with other factors such as inflammation, and altered state of the autonomic nerve system, a history of frequent operations, and emotional stress [2-7]. Recently, there has been an increasing awareness of paroxysmal AF without cardiac or metabolic abnormality which appears to account for $10-20 \%$ of all AF patients $[4,7]$. We describe here a 69-year-old female patient who newly developed AF as a result of a sympatheticparasymapthetic imbalance that might be potentiated by epidural anesthesia for a scheduled open reduction and internal fixation of a right ankle fracture.

Received: October 5, 2009. Revised: 1st, October 27, 2009; 2nd, November 19, 2009. Accepted: December 14, 2009.

Corresponding author: Hye Won Shin, M.D., Department of Anesthesiology and Pain Medicine, College of Medicine, Korea University, 126-1, Anam-dong 5-ga, Seongbuk-gu, Seoul 136-075, Korea. Tel: 82-2-920-5632, Fax: 82-2-928-2275, E-mail: hwshin99@yahoo.com

(c) This is an open-access article distributed under the terms of the Creative Commons Attribution Non-Commercial License (http:// creativecommons.org/licenses/by-nc/3.0/), which permits unrestricted non-commercial use, distribution, and reproduction in any medium, provided the original work is properly cited. 


\section{Case Report}

A 69-year-old, $65 \mathrm{~kg}$, American Society of Anesthesiologists physical status II woman was scheduled to undergo open reduction and internal fixation for a right-sided ankle fracture due to a pedestrian traffic accident. She had a history of bowel obstruction surgery 40 years ago. She had no history of cardiovascular, pulmonary, or respiratory disease. The electrocardiogram (ECG) was unremarkable, but laboratory evaluation revealed low hemoglobin $(9.2 \mathrm{~g} / \mathrm{dl})$ and prolongation of prothrombin time $15.8 \mathrm{sec}$ (74\%, INR 1.23$)$, and chest computed tomography showed bilateral pleural effusion. Echocardiography revealed a good ejection fraction (60\%), mild biventricular hypertrophy, and pulmonary hypertension (40 $\mathrm{mmHg}$ ).

In the previous month, she received 2 surgical operations (closed reduction of left-sided intertrochanteric fracture under general anesthesia and the debridement and irrigation of lower extremity under epidural anesthesia). There were no specific problems or events recorded during these operations.

For the surgery resulting in $\mathrm{AF}$, the patient received $0.5 \mathrm{mg}$ atropine and $2 \mathrm{mg}$ midazolam IM as premedication $30 \mathrm{~min}$ before surgery. Upon arrival in the operating room, an ECG was recorded and automated BP cuff and pulse oximeter $\left(\mathrm{SpO}_{2}\right)$ monitors were applied. The patient's preoperative blood pressure (BP) was 140/70 $\mathrm{mmHg}$ and pre-operative heart rate (HR) was 82 beats/min with a sinus rhythm, and $\mathrm{SpO}_{2}$ was $95 \%$.

She received epidural anesthesia for operation. An 18-gauge Tuohy epidural needle was inserted at L3/4. Loss of resistance was used to identify the epidural space in the left lateral decubitus position. An epidural catheter was inserted through a needle and $3 \mathrm{ml}$ lidocaine $2 \%$ were administered as a test dose, without complications. Via epidural catheter, $9 \mathrm{ml}$ lidocaine $2 \%$ and $50 \mu \mathrm{g}$ fentanyl were given for epidural analgesia. The patient received $500 \mathrm{ml}$ Ringer's solution before and after receiving epidural injection. At fifty minutes after epidural drug injection, the analgesia was obtained up to inguinal area by pinprick test and the BP slowly decreased to $110 / 70 \mathrm{mmHg}$ with HR maintaining 85 beats/min.

Twenty minutes after epidural injection, she was sterilized with disinfectant such as betadine, alcohol for the right-sided leg, but she complained about buttock pain by running down of disinfectant in leg-raising position. At that time, the patient's BP was steady at 110/65 mmHg but her HR abruptly increased to 185 beats/min with a paroxysmal AF pattern (Fig. 1). This paroxysmal AF persisted for $30 \mathrm{~min}$, despite injection of esmolol 30 and digoxin $0.25 \mathrm{mg}$ IV. We thought that the injected dose of epidural anesthetics was not sufficient for pain relief in the leg due to the massive crushing injury of the patient's lower extremity.
We decided to switch the anesthetic method to general anesthesia. The patient was pre-oxygenated, induced with $5 \mathrm{ml}$ propofol $2 \%$ and $0.05 \mu \mathrm{g} / \mathrm{kg} / \mathrm{min}$ remifentanil infusion without muscle relaxant, and was easily mask-ventilated. She was intubated with a 7.0 tracheal tube without incident. Anesthesia was maintained using $2-3 \mathrm{ml} / \mathrm{hr}$ propofol $2 \%$, $0.05 \mu \mathrm{g} / \mathrm{kg} / \mathrm{min}$ remifentanil and $4 \mathrm{~L} / \mathrm{min}$ oxygen immediately after intubation. Five minutes after induction, the patient's BP decreased abruptly to $60 / 40 \mathrm{mmHg}$ while HR continued to be high at 175 beats/min with AF pattern. We decided to cancel the operation because the paroxysmal AF had continued and the patient's vital signs were unstable. All surgical drapes and tourniquets were removed from the operative site, and phenylephrine infusion $0.1-0.5 \mu \mathrm{g} / \mathrm{kg} / \mathrm{min}$ was started. At that time, the BP increased to $115 / 66 \mathrm{mmHg}$ and HR declined to 125 beats/min but the AF pattern sustained. The tracheal intubation was extubated and epidural catheter was removed. The patient regained alert mentality and had $\mathrm{BP}$ of $110 / 70 \mathrm{mmHg}$ and a HR of 120 beats $/ \mathrm{min}$. The duration of anesthesia was $80 \mathrm{mins}$, a total of $600 \mathrm{ml}$ of Hartmann solution was injected, and urine output was $190 \mathrm{ml}$.

The patient was transferred to the intensive care unit and was prescribed diltiazem $30 \mathrm{mg}$ bid (a calcium channel blocker)

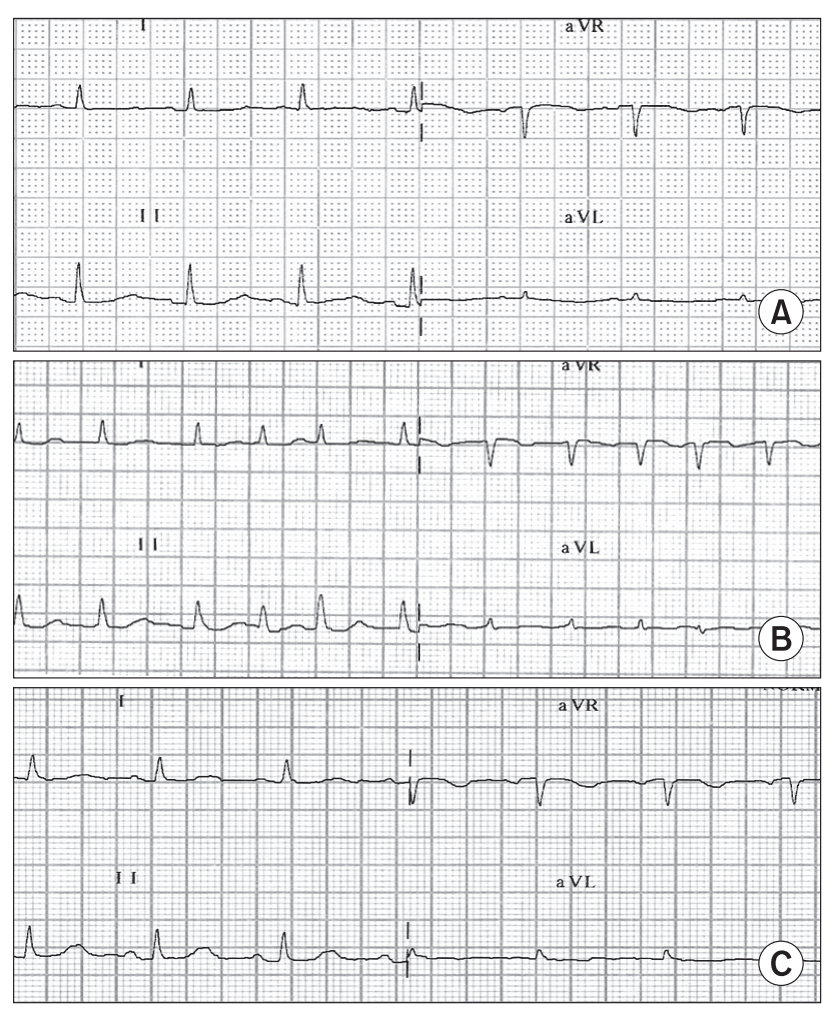

Fig. 1. Electrocardiographic (ECG) findings. (A) Regular sinus rhythm without arrhythmia was observed at preoperative ECG, (B) Atrial fibrillation was developed abruptly after epidural drug injection, (C) ECG returned to normal sinus rhythm in intensive care unit. 
and fraxiparine during following 4 days for thromboembolism prophylaxis. After arriving at the ICU, the patient's HR gradually decreased and the ECG showed normal sinus rhythm with a HR of 81 beats/min. Echocardiography revealed left ventricular hypertrophy, pulmonary hypertension (36 $\mathrm{mmHg}$ ) and normal left ventricular function. On the fourth days after the AF event, the patient underwent surgery for a right-sided intertrochanteric fracture under general anesthesia without any events or problems.

\section{Discussion}

The incidence of perioperative AF in noncardiac, nonthoracic surgical patients is $0.37 \%$, but most frequently occurs within 3 days of surgery $[4,8]$. Perioperative AF is often associated with significant morbidity ( $12 \%$ of AF patients), longer hospital stays due to continued AF ( $16 \%$ of AF patients), and increased costs related to the $\mathrm{AF}[4,8]$. Most instances of $\mathrm{AF}$ are associated with cardiac and some pulmonary diseases as well as metabolic, toxic, endocrine, or genetic abnormalities $[1,9]$, and some AF events are likely related autonomic changes and inflammatory responses [2-6]. Amar [10] suggested several aggravating factors after a review of the literature. These aggravating factor for AF are (i) surgically induced trauma (autonomic denervation, cannulation of atria), (ii) high adrenergic tone (elevated HR before surgery), (iii) inflammatory response (postoperative state, C-reactive protein [CRP], interleukin-6), and (iv) tachycardia (remodeling of the atria, ionic channel changes). Our patient had four aggravation factors (autonomic denervation by epidural anesthesia, high adrenergic tone and tachycardia by light anesthesia, and increased CRP due to inflammation).

Classification of AF subtypes is 'paroxysmal (within 7 days), 'persistent (over 7 days)', and 'permanent' according to the duration of the episode [11]. The most common clinical settings for permanent AF are hypertension and ischemic heart disease. Patients initially presenting with paroxysmal AF often progress to longer, non-self-terminating bouts. Almost half of the patients with paroxysmal AF have no obvious clinical cause (lone or idiopathic AF) [10]. AF without underlying organic abnormalities occurs more often against the background of parasympathetic predominance, whereas AF with structural heart disease usually occurs in a sympathetic setting [12]. AF that is initially responsive to pharmacological treatment or electrical cardioversion tends to become resistant and then cannot be converted to sinus rhythm. Effective prevention is essential in managing this arrhythmia whose occurrence is widespread, progression is relentless, and morbidity and mortality are significant [11].

In our case, a history of frequent operations, inflammation, a state of inadequate analgesia by epidural anesthesia, and surgery-related anxiety may have induced the paroxysmal AF observed. First, the patient received a 3rd operation at postoperative day 2 following the 2 nd operation. Second, inflammation caused by the crushing injury of the patient's lower extremities was evident as her CRP level had increased to $254-449 \mathrm{mg} / \mathrm{L}$ which could increase the possibility of AF development $[2,3]$. Third, the sympathetic and parasympathetic systems are associated with AF, but the increase in acetylcholine by parasympathetic stimulation is known as the main factor involved in the development of AF rather than enhanced vagal or sympathetic drive alone $[5,6,12]$. Sharifov et al. [5] reported on the effects of beta-adrenergic and cholinergic stimulation or blockade on paroxysmal AF by adrenaline or acetylcholine perfusion to the sinus node artery in anesthetized open-chest dogs. Adrenaline and acetylcholine induce AF in 17\% and $100 \%$ of dogs, respectively, and the adrenergic tone facilitated the initiation of cholinergically mediated AF. Their study indicated that although both autonomic systems play a role in the development of AF, cholinergic stimulation is the primary factor for paroxysmal AF initiation while adrenergic tone facilitates the initiation and maintenance of cholinergically mediated AF. In our case, parasympathetic predominance induced by epidural sympathetic blockade might induce the development of AF, and the increase in adrenergic activity from pain due to insufficient anesthesia facilitated the initiation and maintenance of AF. In a recent study, a primary role of cholinergic stimulation in AF generation was made evident by revealing an abrupt shift toward vagal predominance immediately before AF onset in patients with paroxysmal AF [12]. Lastly, approximately one third of AF patients have elevated levels of depression and anxiety [7]. In our case, the preoperative surgery-related emotional stress of the patient facilitated the development of AF.

Generally, the purposes of AF treatment are (i) to control $\mathrm{HR}$, (ii) to maintain of sinus rhythm, and (iii) to decrease the thromboembolism risk [13]. The drugs used for HR control are $\beta$-blocking drugs, calcium channel blockers, and digoxin, and electrical cardioversion is used to treat hemodynamically unstable AF. In our case, esmolol $30 \mathrm{mg}$ and digoxin $0.25 \mathrm{mg}$ were used, but AF continued. The combined use of digoxin and esmolol is usually effective in the control of HR. The onset of digoxin takes up to $30 \mathrm{~min}$ and the peak effect is obtained at $1-4$ hr. By the delayed effect of this drug, AF slowly disappeared [14].

When AF persists without a conversion of sinus rhythm, the potential for thromboembolism develops within $48 \mathrm{hr}$. If AF is seen to cause a greater risk of stroke [13], then heparin must be used prophylactically.

Once AF is controlled in the perioperative period, the anesthesiologist is then faced with the dilemma of whether to proceed or delay the surgery. Although the incidence of newly 
developed atrial arrhythmias associated with acute myocardial infarction, pulmonary embolism, pericarditis, or thyrotoxicosis is less than $1 \%$, physicians need to rule out significant structural heart disease using transthoracic echocardiogram in making their decision on how to proceed [15].

In conclusion, paroxysmal AF in elderly patients is not an unusual arrhythmia. During anesthetic management, the proper preoperative prevention and intraoperative treatment are needed in patients at high risk for paroxysmal AF.

\section{References}

1. Alpert JS, Petersen P, Godtfredsen J. Atrial fibrillation: natural history, complications, and management. Ann Rev Med 1988; 39: 41-52.

2. Aviles RJ, Martin DO, Apperson-Hansen C, Houghtaling PL, Rautaharju P, Kronmal RA, et al. Inflammation as a risk factor for atrial fibrillation. Circulation 2003; 108: 3006-10.

3. Hatzinikolaou-Kotsakou E, Tziakas D, Hotidis A, Stakos D, Floros D, Papanas N, et al. Relation of C-reactive protein to the first onset and the recurrence rate in lone atrial fibrillation. Am J Cardiol 2006; 97 : 659-61.

4. Brathwaite D, Weissman C. The new onset of atrial arrhythmias following major noncardiothoracic surgery is associated with increased mortality. Chest 1998; 114: 462-8.

5. Sharifov OF, Fedorov VV, Beloshapko GG, Glukhov AV, Yushmanova AV, Rosenshtraukh LV. Roles of adrenergic and cholinergic stimulation in spontaneous atrial fibrillation in dogs. J Am Coll Cardiol 2004; 43: 483-90.

6. Tomita T, Takei M, Saikawa Y, Hanaoka T, Uchikawa S, Tsutsui H, et al. Role of autonomic tone in the initiation and termination of paroxysmal atrial fibrillation in patients without structural heart disease. J Cardiovasc Electrophysiol 2003; 14: 559-64.

7. Thrall G, Lip GY, Carroll D, Lane D. Depression, anxiety, and quality of life in patients with atrial fibrillation. Chest 2007; 132: 1259-64.

8. Christians KK, Wu B, Quebbeman EJ, Brasel KJ. Postoperative atrial fibrillation in noncardiothoracic surgical patients. Am J Surg 2001; 182: 713-5.

9. Brugada R, Tapscott T, Czernuszewicz GZ, Marian AJ, Iglesias A, Mont L, et al. Identification of a genetic locus for familial atrial fibrillation. N Engl J Med 1997; 336: 905-11.

10. Amar D. Perioperative atrial tachyarrhythmias. Anesthesiology 2002; 97: 1618-23.

11. Allessie MA, Boyden PA, Camm AJ, Kléber AG, Lab MJ, Legato MJ, et al. Pathophysiology and prevention of atrial fibrillation. Circulation 2001; 103: 769-77.

12. Fioranelli M, Piccoli M, Mileto GM, Sgreccia F, Azzolini P, Risa MP, et al. Analysis of heart rate variability five minutes before the onset of paroxysmal atrial fibrillation. Pacing Clin Electrophysiol 1999; 22: 743-9.

13. Peters NS, Schilling RJ, Kanagaratnam P, Markides V. Atrial fibrillation: strategies to control, combat, and cure. Lancet 2002; 359: 593603.

14. Fujiki A, Sakabe M, Nishida K, Mizumaki K, Inoue H. Role of fibrillation cycle length in spontaneous and drug-induced termination of human atrial fibrillation. Circ J 2003; 67: 391-5.

15. Fuster V, Rydén LE, Asinger RW, Cannom DS, Crijns HJ, Frye RL, et al. ACC/AHA/ESC guidelines for the management of patients with atrial fibrillation: executive summary a report of the American College of Cardiology/American Heart Association task force on practice guidelines and the European Society of Cardiology Committee for practice guidelines and policy conferences (Committee to develop guidelines for the management of patients with atrial fibrillation) developed in collaboration with the North American Society of Pacing and Electrophysiology. Circulation 2001; 104: 2118-50. 